\title{
Using iNaturalist to Contribute Your Nature Observations to Science ${ }^{1}$
}

\author{
Matthew Earl Boone and Mathieu Basille ${ }^{2}$
}

\section{Abstract}

Citizen science allows everyday citizens to contribute time and data to scientific projects. iNaturalist is one of the most popular citizen science data portals in the world. At iNaturalist, people who have observed interesting phenomena in nature can submit pictures of their observations to an online database. A growing online community of citizens and experts reviews the submitted observations and makes identifications. The data are used for important biodiversity research around the world. In addition, the data portal serves as a hub for users to plan community projects and learn more about species identification and biodiversity. iNaturalist is easy to get started with by simply signing up online at https://www.inaturalist.org or by downloading the iNaturalist mobile app. The number of users on iNaturalist hovers around 500,000, and yet concise literature on iNaturalist's functionality remains sparse. In this publication, we explain the history of iNaturalist and how observations are vetted and used, and we include a step-by-step guide on how to get started with iNaturalist.

\section{Introduction}

Citizen-submitted data collection is a rapidly expanding way for citizen scientists to actively engage with the natural world, and it has become a popular tool for researchers. More and more citizens submit their nature observations to online databases, which in turn contribute to research across the world in biodiversity, climate change, and invasive species conservation (Chandler et al. 2017; Follett and Strezov 2015). Citizens' observations contribute to important research, and scientists gain access to a larger data collection network. Popular programs like iNaturalist and eBird allow users to input their sightings of the natural world on their phones, and local experts help guide identifications of observations, engaging users further and creating communities. iNaturalist is the most popular general-purpose biodiversity portal. It uses a community-driven model to identify and vet observations, wherein communities of experts and volunteers agree on identification of observations, allowing even amateur naturalists to contribute data without needing to be an expert. iNaturalist was created in 2008 to embrace citizen science data collection of biodiversity observations across the world and to help people learn about nature. Created originally as a collaboration between three graduate students at University of California Berkley, it was later funded by the California Academy of Sciences in 2014 and National Geographic Society in 2017. To date, iNaturalist contains almost 13 million individual records of species ranging from fungi, plants, insects, and animals. iNaturalist has been used to study the spread of invasive species (Creley and Muchlinski 2017), the presence of rare or hard-to-sample species (Michonneau and Paulay 2015), and new occurrences of species across the world. Teachers and nature centers can use iNaturalist to teach students about topics including wildlife identification, climate change, and the limitations of machine learning.

1. This document is WEC413, one of a series of the Department of Wildlife Ecology and Conservation, UF/IFAS Extension. Original publication date June 2019. Visit the EDIS website at https://edis.ifas.ufl.edu for the currently supported version of this publication.

2. Matthew Earl Boone, biological scientist Il; and Mathieu Basille, assistant professor of landscape ecology; https://edis.ifas.ufl.edu, UF/IFAS Ft.Lauderdale Research and Education Center, Davie, FL 33314.

The Institute of Food and Agricultural Sciences (IFAS) is an Equal Opportunity Institution authorized to provide research, educational information and other services

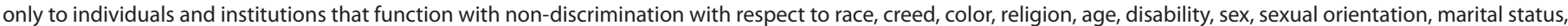

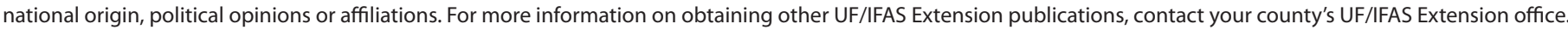
U.S. Department of Agriculture, UF/IFAS Extension Service, University of Florida, IFAS, Florida A \& M University Cooperative Extension Program, and Boards of County Commissioners Cooperating. Nick T. Place, dean for UF/IFAS Extension. 


\section{How iNaturalist Works}

iNaturalist works by accepting user-collected presence data on individual species found, which is called an "observation." Observations are singular, spatially, and temporally referenced data points uploaded by a single user. Users can suggest their own identification of the specimen; however, every observation is subject to community vetting on the identification. Because of this, iNaturalist strongly recommends uploading photos or audio of species as supporting material. Users can input observations through their phones (the easiest method) or through the online portal, after which observations are entered into the iNaturalist database. While observations are generally entered singularly, a bulk option is available on the data portal.

When users upload a picture, iNaturalist attempts to identify and make suggestions about the identification of the specimen using their newly integrated machine learning software (Van Horn et al. 2015). This software uses local observations to weight the likelihood of identifications and give suggestions on possible species identifications. With each suggestion, iNaturalist links information like taxonomy and species descriptions (provided by Wikipedia) to help guide the user's decisions. Users can enter their best guess of the identification or simply select "unknown" and allow the community to identify the observation. Ultimately, this allows any user, beginners and experts alike, to contribute to data collection without having to know anything specific about identification of that taxa.

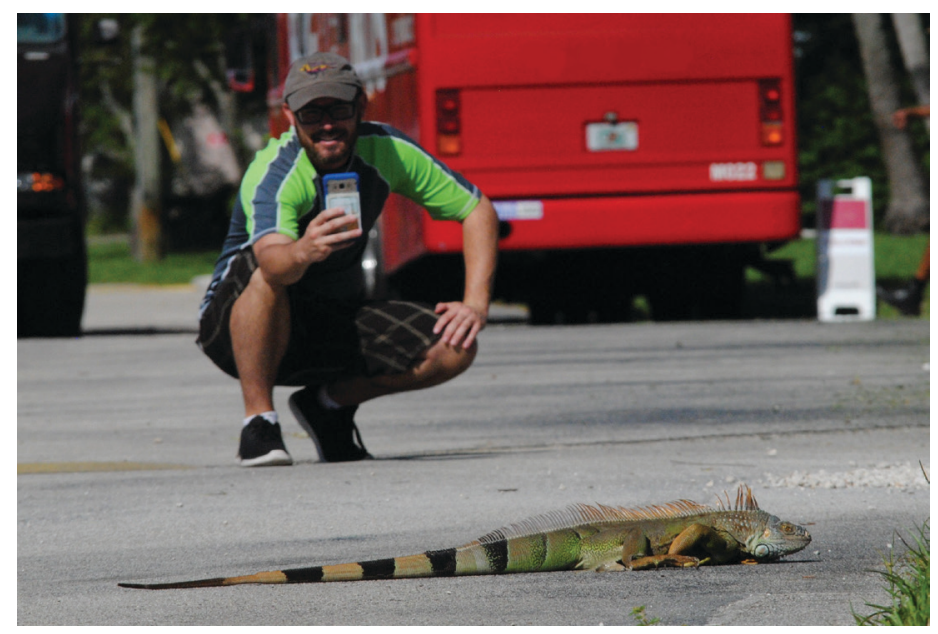

Figure 1. iNaturalist allows you to upload pictures of your daily nature observations to the iNaturalist database. These observations are added to the 13 million other user-submitted observations around the world.

Credits: Simona Picardi. Creative Commons License

\section{Data Validation}

Once uploaded to the database, observations are then validated through a community identification effort. Each photo sample can be reviewed by any registered user on the website. Users across the iNaturalist community attempt to identify each observation down to the lowest taxonomic hierarchy possible. iNaturalist separates observations into two categories: "Casual" and "Research Grade." Research Grade observations are the highest level of data quality and enter into the scientific database that is free to use for research purposes. To be considered "Research Grade," observations must meet four initial criteria. They must include a date, a spatial georeference, and a picture/or sound, and the subject must be a naturally living organism (not captive or cultivated). Observations without visual or audio evidence are "Casual Grade" and do not enter the research database, stressing further the importance of entering photos or audio samples in iNaturalist. The first three criteria are easily produced with most cellphones, whose cameras typically record both the timestamp and the spatial coordinates using the GPS on board.

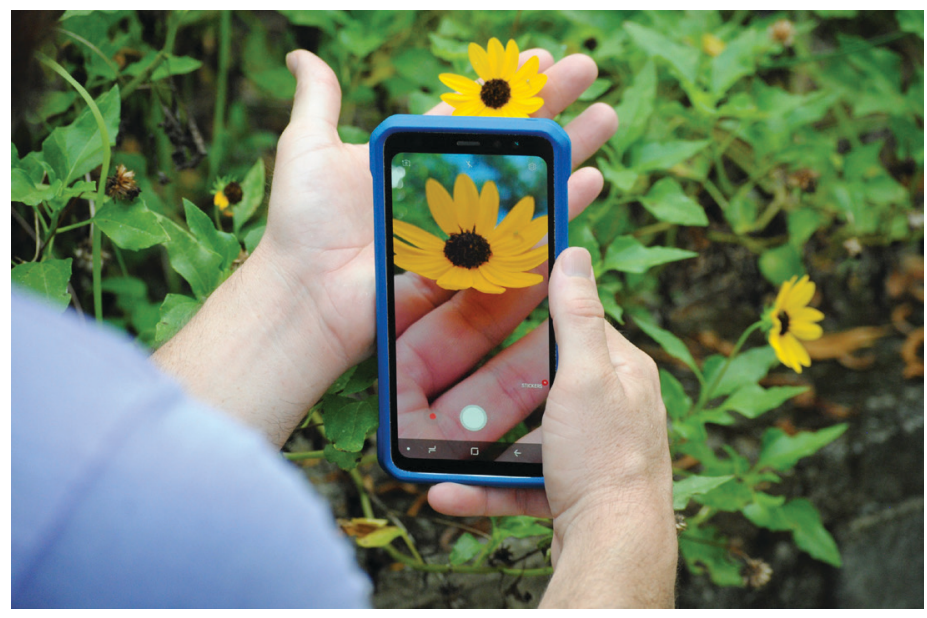

Figure 2. You can upload pictures directly from your phone using the iNaturalist app. Once uploaded the photos are automatically georeferenced and possible identifications are suggested via iNaturalist's newly integrated machine learning software. Credits: Simona Picardi. Creative Commons License

Identifications then go through the community vetting process that requires at least 2 out of 3 additional users to agree on the identity of a specimen. iNaturalist attempts to facilitate discussion between users by weighting identifications higher that are contrary to the leading guess. The lowest level of taxonomy that reaches this threshold becomes the accepted identification. For example, say someone uploads a picture of a large black bird. Four users agree it is some type of grackle (Genus Quiscalus), but two of them think it is a common grackle (Quiscalus quiscula) while the other two think it is a boat-tailed grackle (Quiscalus major). Because neither observation reaches the $2 / 3$ agreement at 
the species level, the higher genus level is accepted (agreed on by $4 / 4$ users), and the observation gets identified in the genus Quiscalus. If later users agree on the species level identification above the $2 / 3$ threshold, then the submission is reentered as that species. An observation that reaches this threshold can be considered Research Grade and entered into the Global Biodiversity Information Facility (GBIF) database. This international collaboration collates biodiversity records from scientific experts worldwide and nowadays also includes citizen-science databases like iNaturalist, Breeding Bird Surveys, and eBird. At the GBIF website (https://www.gbif.org/), any user can download the gathered occurrence data for personal or research purposes (Edwards, Lane, and Nielsen 2000)

To help users better identify observations, iNaturalist allows uploading of what they call "Guides." These are online guide books of species in particular regions or taxa users expect to encounter. They can be tailored to individual projects ("Birds of the Everglades BioBlitz") or to species and regions ("Noctuid Moths of South Florida"). This tool allows the community to steer users towards likely identifications and teaches users about new things to look for as they explore. Anybody can create and publish a "Guide" using the iNaturalist online tool and later share it with a fixed URL they can share.

In addition to online guides, the website allows users to browse through the global database and identify others' observations. From there a user can help to identify specimens or learn key features in identifying taxa. iNaturalist attempts to foster a friendly and active online community to help identify observations regardless of skill level. They do this by tracking users' participation in identifying other observations and encouraging experts to include descriptions of how they identified specimens. The online community is strengthened by a strict adherence to their broad community guidelines (https://www.inaturalist.org/ pages/community+guidelines). Hate speech, insults, and harassment are strictly forbidden, and iNaturalist openly bans users who discriminate in any manner. More nuanced suggestions include not using all caps, avoiding sarcasm, and not justifying identifications using your credentials. iNaturalist's strict core rules and broad suggestive guidelines help to cultivate a helpful and accepting online community that is vital to the citizen science model. In turn, the community helps police errant identifications and identify malicious users.

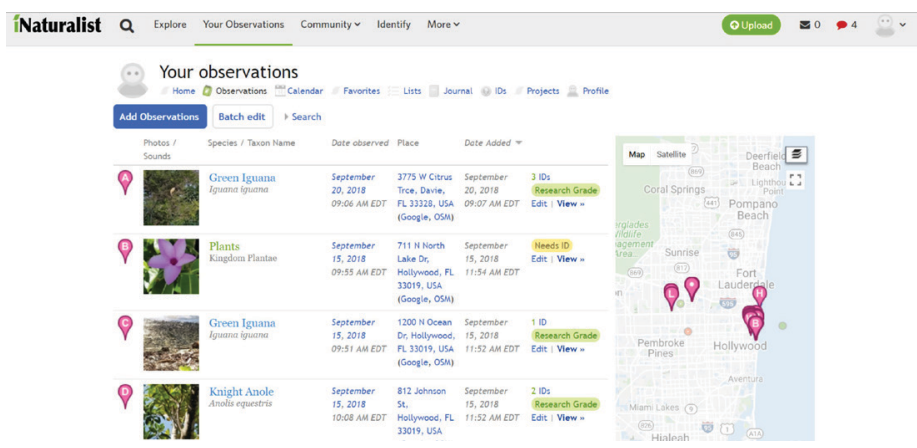

Figure 3. The iNaturalist website collates your observations, tracks your statistics, and allows users to submit suggestions on the identification of your observation.

Credits: http://www.inaturalist.org

\section{Projects and BioBlitzes}

iNaturalist encourages groups working on projects to use the system to facilitate data collection.. In iNaturalist, "Projects" is an umbrella term intended to capture or enhance specific categories of observations. Simple projects can be a "Collection," i.e., a collection of all observations with certain criteria. Collections act as filters for observations and can assist a project manager to more easily track and collect certain types of data (for instance creating a project for a state park, or on moths of Broward county). A "Traditional" project allows more complicated data collection options than simple observations. In a traditional project, group administrators can ask for data that is not normally collected, like the approximate area of an invasive species outbreak or body condition of the species in question.

The most popular use of iNaturalist Projects is using iNaturalist for BioBlitzes, which are intensive citizenscience biodiversity surveys (see the publication BioBlitzes: Citizen Science for Biodiversity in Florida). This allows even inexperienced users to log observations for the BioBlitz and allows BioBlitz organizers to use iNaturalist as a convenient data portal. The BioBlitz Project allows coordinators to interact with the participants about scheduling and provide memos, and it allows participants to see the results of their efforts in near-real time. To use iNaturalist in BioBlitz, the project manager creates a project for the BioBlitz and creates a spatial boundary for the project. The URL for the event can be shared to participants, giving additional information and a website for the project. On the day of the BioBlitz, individual participants actively upload their photos via the phone app; observation locations will be recorded as long as the users' location services are turned on. Section leaders for the BioBlitz can submit the results online afterwards using the bulk import tool. This way, even species without photos can be counted for the BioBlitz totals. Once georeferenced, the BioBlitz can summarize all observations within the spatial boundary. 


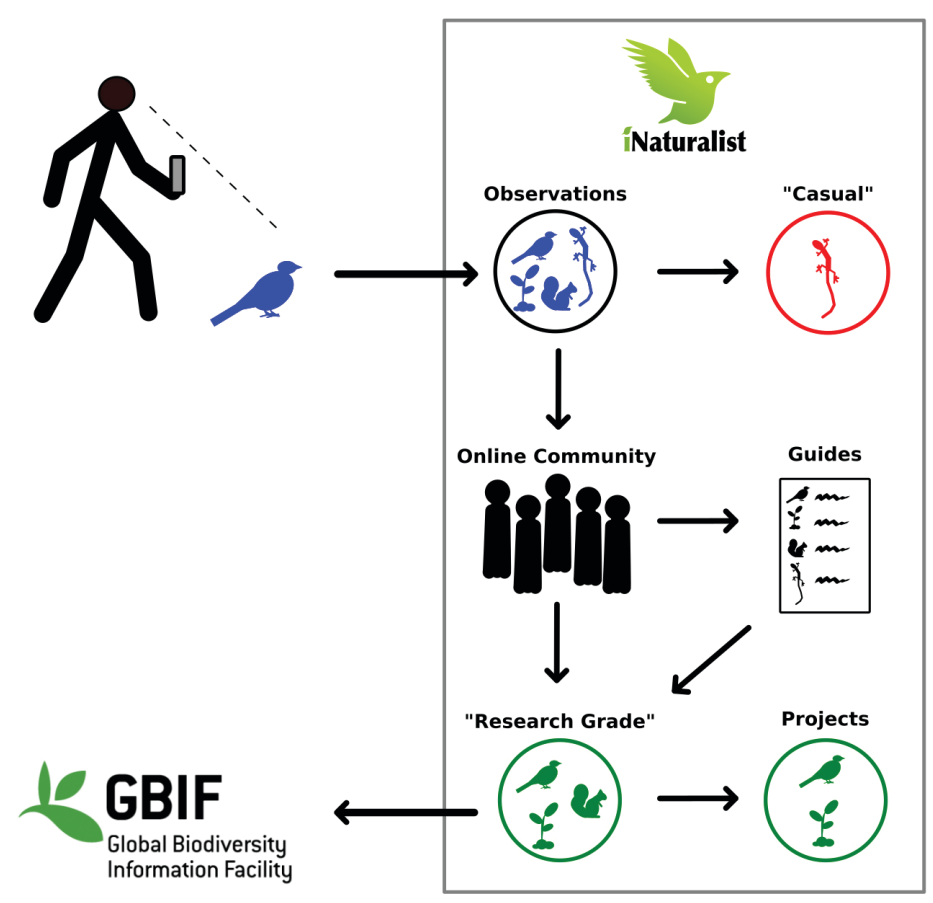

Figure 4. Users upload their observations to the web portal (usually through their phones). These observations are identified and vetted by the online community becoming Research Grade observations. Users create Guides to help other users identify species. Observations that arent validated are considered Casual observations. Research Grade observations are eventually uploaded into the GBIF database and used by Projects within iNaturalist.

\section{Getting started with iNaturalist}

The easiest way to get started with iNaturalist is to sign up for iNaturalist and download the app. You can sign up for iNaturalist on the website or when you download the app. The process requires only an email, and a user name and password are chosen by the user. The app can be freely downloaded on Android or iPhone in their respective app stores.

Submitting an observation is relatively straightforward on the app. When you find something interesting, you can either take a picture first and then upload it on the app, or take a picture directly within the app. If you take a picture outside of the app, once uploading the picture to the app you must hit the "Location" button to "Get" your current location or "Edit" to manually select a location. This is important because to get research grade status, observations must be georeferenced. After uploading the photo, you can go to "What did you see" and attempt to identify the specimen using iNaturalist's suggestions, or you may leave it "Unknown." Even if you are not able to identify the specimen, it is best to give it a broad taxa name like "Plants" or "Aves" or "Reptiles" before submitting. This allows the observation to be sorted so that the online community can more easily find it to identify.
If you are in an area without cell phone service or Wi-Fi, then the observations will be stored locally on your phone, requiring you to hit "Sync" on the app when you get service to push observations to the website. For further information, there is a plethora of online user guides for download, use, and navigation of iNaturalist (https://www.inaturalist. org/pages/help).

Submitting your daily nature observations to iNaturalist is a fun and easy way to contribute to biodiversity research. iNaturalist's robust apps and supportive online community facilitate interactions between users and build a dedicated community that is vital to large-scale citizen science projects. As iNaturalist's user base grows, so, too, the uses for this important data set will grow. This makes it an increasingly vital tool for biodiversity research and a novel outreach tool for educators and scientists.

\section{References}

Chandler, M., L. See, K. Copas, A. M. Z. Bonde, B. C. López, F. Danielsen, J. Kristoffer et al. 2017. "Contribution of citizen science towards international biodiversity monitoring." Biological Conservation 213: 280-294. https://doi. org/10.1016/j.biocon.2016.09.004

Creley, C. M., and A. E. Muchlinski. 2017. “Distribution of the Eastern Gray Squirrel (Sciurus carolinensis) within California as of 2015." Bulletin, Southern California Academy of Sciences, 116 (3): 204-213. https://doi.org/10.3160/ soca-116-03-204-213.1

Edwards, J. L., M. A. Lane, and E. S. Nielsen. 2000. "Interoperability of Biodiversity Databases : Biodiversity lnformation on Every Desktop." Science 289 (September): 2312-2314. https://doi.org/10.1126/science.289.5488.2312

Follett, R., and V. Strezov. 2015. An analysis of citizen science based research: Usage and publication patterns. PLoS ONE 10 (11): 1-14. https://doi.org/10.1371/journal. pone. 0143687

Michonneau, F., and G. Paulay. 2015. "Using iNaturalist to learn more about echinoderms." Reef Encounter 30 (1): 29-31. 
Van Horn, G., S. Branson, R. Farrell, S. Haber, J. Barry, P. Ipeirotis, P. Perona et al. 2015. "Building a bird recognition app and large scale dataset with citizen scientists: The fine print in fine-grained dataset collection." Proceedings of the IEEE Computer Society Conference on Computer Vision and Pattern Recognition, 07-12-June, 595-604. https://doi. org/10.1109/CVPR.2015.7298658 